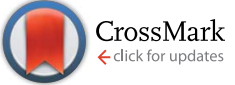

Cite this: RSC Adv., 2017, 7, 5920
Received 24th October 2016 Accepted 22nd November 2016 DOI: 10.1039/c6ra25711a www.rsc.org/advances

\title{
A rapid ion chromatography column-switching method for online sample pretreatment and determination of L-carnitine, choline and mineral ions in milk and powdered infant formula $\dagger$
}

\begin{abstract}
Dan Wei, ${ }^{\text {a }}$ Xu Wang, ${ }^{\mathrm{b}}$ Nani Wang ${ }^{\mathrm{c}}$ and Yan Zhu*a
A simple and rapid column-switching ion chromatography with non-suppressed conductivity detection method was developed for simultaneous determination of L-carnitine, choline and mineral ions (free sodium, potassium, magnesium and calcium ions) in milk and powdered infant formula samples. On-line sample clean-up was achieved on the pretreatment column Dionex lonPac NG1 $(50 \mathrm{~mm} \times 4 \mathrm{~mm}$, i.d.), with pretreatment elution ( $\left.3 \mathrm{mmol} \mathrm{L}^{-1} \mathrm{MSA}\right)$ and washing solution (100\% acetonitrile) at a flow rate of $1.0 \mathrm{~mL} \mathrm{~min}{ }^{-1}$. After column switching, the separation and determination of analytes were performed using guard column Dionex lonPac SGS $(50 \mathrm{~mm} \times 4 \mathrm{~mm}$, i.d.) and analytical column Dionex lonPac SCS (250 $\mathrm{mm} \times 4 \mathrm{~mm}$, i.d.) with an isocratic mobile phase consisting of $3 \mathrm{mmol} \mathrm{L}{ }^{-1} \mathrm{MSA}$ and $10 \%(\mathrm{v} / \mathrm{v})$

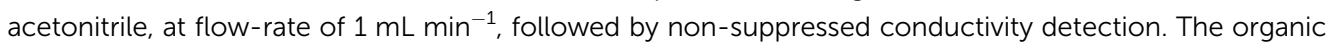
matrices clean-up performance of the proposed method was studied using an UV detector working at 200-400 $\mathrm{nm}$. Under the optimal conditions, detection limits $(\mathrm{S} / \mathrm{N}=3)$ ranging from $0.05-0.30 \mathrm{mg} \mathrm{L}^{-1}$, good linearities in the range of $1-100 \mathrm{mg} \mathrm{L}^{-1}$ with a correlation coefficient over 0.9973 , and satisfactory recoveries in the range between $94 \%$ and $105 \%$ with relative standard deviation less than $2.1 \%$ for real samples were attainted. The proposed method was successfully applied to rapid, simultaneous, and labour-saving detection of L-carnitine, choline, and mineral ions in milk and powdered infant formula samples.
\end{abstract}

\section{Introduction}

Infant formula has been recognized as a very important dairy product regarding the wide consumption for children under 1 year. The composition of infant formula, especially L-carnitine, choline, and mineral ions (free sodium, potassium, magnesium and calcium ions), play essential roles in both optimal growth (e.g. bone, brain and visual development) and the development of a newborn's immune system against an array of diseases and infections..$^{1-3}$ In detail, L-carnitine, a critical substance needed for the maintenance of health, is used as a carrier to transport long-chain fatty acids into the mitochondria of a cell for betaoxidation to produce energy. Carnitine deficiency may lead to insufficient transport of fatty acids through the mitochondrial membrane therefore impairing the mitochondrial energy

${ }^{a}$ Department of Chemistry, Zhejiang University, Xixi Campus, Hangzhou, Zhejiang, China. E-mail: zhuyan@zju.edu.cn

${ }^{b}$ Department of Medical Laboratory, Hangzhou Medical College, Hangzhou, Zhejiang, China

'Department of Medicine, Zhejiang Academy of Traditional Chinese Medicine, Hangzhou, Zhejiang, China

$\dagger$ Electronic supplementary information (ESI) available. See DOI: $10.1039 / \mathrm{c} 6 \mathrm{ra} 25711 \mathrm{a}$

metabolism. Choline is essential for the normal function of all cells. Adequate choline intake is very important for fetal development, memory function, bone formation, normal liver and kidney functions and the prevention of various diseases. ${ }^{4-7}$ The mineral ions are the main cations in milk and their fraction contributes to the nutritional quality of milk and largely determines its physicochemical state, thereby determining the conformation and stability of milk proteins. ${ }^{8}$ More specifically, sodium is the principal cation in extracellular fluid, which is involved in nerve and muscle function, regulation of plasma volume and acid-base balance. ${ }^{9}$ Potassium, as the major cation in intracellular fluid, plays an important role in the regulation of osmotic pressure, blood pressure and acid-base balance. ${ }^{\mathbf{1 0}}$ Magnesium is primarily an intracellular nutrient and plays roles in protein and carbohydrate metabolism, synthesis of DNA, and muscle relaxation. Furthermore, magnesium forms a complex with ATP, which serves as the true substrate for biochemical reactions involving energy utilization. Calcium is important in the development and maintenance of strong bones and teeth and also involved in nerve function, muscle contraction and blood clotting. ${ }^{11}$ The excess or absence of these nutritional elements can also cause several health problems. Thus, it is necessary to develop a simple and rapid method for these 
nutritional elements determination in milk and powered infant formulas to ensure rigorous control of their composition, including elements added routinely in order to satisfy such nutrients content requirements.

Numerous methods for analysis of $\mathrm{L}$-carnitine, choline, and mineral ions (free sodium, potassium, magnesium and calcium ions) either alone or in various combinations have been developed, including gas chromatographic (GC) ${ }^{\mathbf{1 2}}$ and conventional HPLC and ion chromatography (IC). The limitation of GC analysis is the need to conversion of choline into a volatile derivative. The conventional HPLC with different detectors, such as evaporative light scattering and mass spectrometry detection, ${ }^{\mathbf{1 3}}$ electrochemical detection and, ${ }^{\mathbf{1 4}}$ suppression conductivity detection ${ }^{\mathbf{1 5}}$ has restrictions in terms of weak chromophore, derivatization and redissolution of the extract in organic solvent. Also, IC with conductivity detection has been evaluated for the separation and determination of choline, ${ }^{16}$ L-carnitine ${ }^{17}$ sodium, potassium, magnesium and calcium ions ${ }^{18,19}$ in food samples ${ }^{20,21}$ and is an attractive alternative. It is well known that IC is a powerful analytical technique for the separation of cations, anions and polar and hydrophilic compounds. However, the direct determination of the target analytes in milk samples is difficult. Complex matrices, including proteins, lipids, carbohydrates, vitamins, minerals and enzymes, would damage the IC columns, decrease sensitivity, selectivity, and reproducibility. Solidphase extraction (SPE) is the most commonly used methods for clean-up and extraction, ${ }^{22,23}$ and usually carried out in offline mode. Using these sample pretreatment methods could be accompanied with risk of analytes losses during sample manipulation and greater organic hazard. Recent application of column-switching technique for on-line extraction is the growing trend in sample preparation procedures. ${ }^{24}$ Columnswitching technique using special sorbents for complex matrix samples can work without the laborious and timeconsuming clean-up protocols. From this perspective, column-switching technique for direct injection, on-line preparation and simultaneously analysis of samples is modern approach in implementation of all of analytical processes to one step. The main advantage of this technique is strong reduction of time and sample manipulation during sample preparation steps. Moreover, its application has expanded to the analysis of honeydew and nectar, Colla corii asini. ${ }^{25,26}$ However, there are few applications in quantification of nutrients in milk products.

In this paper, we presents a rapid on-line column-switching IC method for simultaneous determination of L-carnitine, choline, sodium, potassium, magnesium and calcium ions in milk and powdered infant formula samples. IonPac NG1, a kind of polymer-based reversed-phase C-18 column, was used as precolumn. This column could enable direct injection of the samples into the flow analytical systems, because it would absorb organic interferences to the hydrophobic part of the stationary phase and, at the same time, exclude target ionic molecules. The proposed method was developed and validated and may have useful applications in milk products quality control.

\section{Materials and methods}

\subsection{Instrumentation}

Chromatographic analysis was performed on ICS-3000 (ThermoFisher Scientific Waltham, MA, USA) consisting of two quaternary pumps with automated membrane eluent degassing, a column heater, two six port valves. On-line sample pretreatments were carried out using Dionex IonPac NG1 guard column (50 $\mathrm{mm} \times 4 \mathrm{~mm}$, i.d.). Separations were accomplished using a Dionex IonPac SCS (250 $\mathrm{mm} \times 4 \mathrm{~mm}$, i.d.) analytical column and IonPac SGS (50 $\mathrm{mm} \times 4 \mathrm{~mm}$, i.d.) guard column. The separated cations were determined by non-suppressed conductivity detection; an UV detector (UVD 340U, Thermo-Fisher Scientific Waltham, MA, USA) was applied in validation experiment. $25 \mu \mathrm{L}$ of prepared sample was injected into the loop of the chromatograph and a $1 \mathrm{~mL}$ polyether ether ketone tube used as a collection loop. Polyether ether ketone (PEEK) tubes with the lengths as short as possible were used to connect all chromatographic

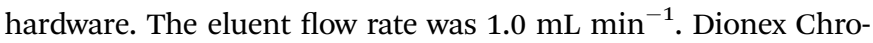
meleon 6.8 software (Thermo-Fisher Scientific Waltham, MA, USA) was used for instrument control and data handling.

A SBL-5DTS thermostatic sonic water bath (Scientz biotechnology Co., LTD, Ningbo, China) was used to control the temperature and sonicate the samples. A centrifuge model LG16-B (Lab centrifuge Co., LTD, Beijing, China) was utilized for the centrifugation.

\subsection{Reagents and samples}

Choline chloride and L-carnitine was purchased from SigmaAldrich (St. Louis, MO, USA). $1000 \mathrm{mg} \mathrm{mL}^{-1}$ of sodium, potassium, magnesium and calcium were obtained from National Research Center for Certified Reference Materials (Beijing, China). Deionized water employed in all experiments was obtained from a Genpure water system (TKA, Niederelbert, Germany), with resistivity not lower than $18.2 \mathrm{M} \Omega \mathrm{cm}$. Extra pure methanesulfonic acid (MSA) and acetonitrile of HPLC grade was obtained from ThermoFisher Scientific (Waltham, MA, USA). All the reagents used in this study were of analytical-reagent grade. Six real samples from six different manufactures were purchased from local supermarket in Hangzhou, China, and stored at $4{ }^{\circ} \mathrm{C}$ until used in this study.

\subsection{Preparation of the standard solutions and spiked milk samples}

Stock solutions containing $1000 \mathrm{mg} \mathrm{L}^{-1}$ of L-carnitine and chloride were prepared by dissolving of substances using deionized water. The stock solutions were kept at 0 to $4{ }^{\circ} \mathrm{C}$ for no longer than one month. The stock solution was further diluted with deionized water to obtain working standard solutions used for method development and validation. The calibration standard solutions were prepared at seven concentration levels $(1,2,5,10,20,30,50$, 75 and $100 \mathrm{mg} \mathrm{L}^{-1}$ ) for the establishment of the calibration curve. All solutions were sealed and kept at 0 to $4{ }^{\circ} \mathrm{C}$ for no longer than one week before analysis.

Prior to analysis, $1 \mathrm{~g}$ of milk powder was accurately weighed and transferred to a $250 \mathrm{~mL}$ flask, to which $8 \mathrm{~mL}$ of deionized 
water was added, and the solution was stirred to dissolve the powder, ultrasonicated for $10 \mathrm{~min}$ at $70{ }^{\circ} \mathrm{C}, 40 \mathrm{~Hz}$ and cooled down to the room temperature. Then, $24 \mathrm{~mL}$ of acetonitrile was added, centrifuged at $10000 \mathrm{rpm}$ for $10 \mathrm{~min}$. The supernatant solutions were diluted 5 times with deionized water and filtered through a $0.45 \mu \mathrm{m}$ nylon filter before IC analysis. In the case of liquid milk, $1 \mathrm{~mL}$ was transferred to $10 \mathrm{~mL}$ centrifugal tube, to which $3 \mathrm{~mL}$ of acetonitrile was added, and then centrifuged at $10000 \mathrm{rpm}$ for $10 \mathrm{~min}$. The supernatant solutions were diluted 10 times with deionized water and filtered through a $0.45 \mu \mathrm{m}$ nylon filter before IC analysis.

The spiked samples were prepared by spiking the proper amounts of each working solution into $1.0 \mathrm{~mL}$ milk or milk powder as prepared above. Samples with higher concentration were diluted with deionized water to obtain concentration inside calibration range. All solutions were stored at $4{ }^{\circ} \mathrm{C}$ before analysis.

\subsection{IC column switching analysis}

The simultaneous on-line sample clean-up and determination was performed using a two columns system. The sample pretreatment column was IonPac NG1 guard column $(50 \mathrm{~mm}$ $\times 4 \mathrm{~mm}$, i.d.), with pretreatment elution ( $\left.3 \mathrm{mmol} \mathrm{L}^{-1} \mathrm{MSA}\right)$ and washing solution ( $100 \%$ acetonitrile) at a flow rate of $1.0 \mathrm{~mL} \mathrm{~min}^{-1}$. The chromatographic separation was performed on an analytical column Dionex IonPac SCS
(250 $\mathrm{mm} \times 4 \mathrm{~mm}$, i.d.) and guard column Dionex IonPac SGS (50 $\mathrm{mm} \times 4 \mathrm{~mm}$, i.d.), using isocratic mobile phase consisting of $3 \mathrm{mmol} \mathrm{L}^{-1} \mathrm{MSA}$ and $10 \%(\mathrm{v} / \mathrm{v})$ acetonitrile and flow rate of $1 \mathrm{~mL} \min ^{-1}$. Column temperature was kept at $30{ }^{\circ} \mathrm{C}$ for both columns.

Fig. 1 shows the IC column switching procedures: (1) loading sample (Fig. 1A), (2) on-line sample clean-up pretreatment (Fig. 1B), (3) analyzing and rinsing the pretreatment column (Fig. 1C), and (4) analyzing and the pretreatment column achieving the equilibrium (Fig. 1C). First, $25 \mu \mathrm{L}$ volume of sample was loaded into the sample loop (Fig. 1A), and then injected into the IonPac NG1 guard column with washing mobile phase of $3 \mathrm{mmol} \mathrm{L}^{-1} \mathrm{MSA}$ at the flow-rate of $1 \mathrm{~mL} \mathrm{~min}^{-1}$ generated by one of the quaternary pumps (Fig. 1B). This condition aided six analytes rapidly eluted and removal of most organic components from samples in the IonPac NG1 guard column. After being completely collected, the analytes in the $1 \mathrm{~mL}$ collection loop were sent to the analytical columns for separation with washing mobile phase of $3 \mathrm{mmol} \mathrm{L}^{-1} \mathrm{MSA}$ containing $10 \%$ acetonitrile at the flow-

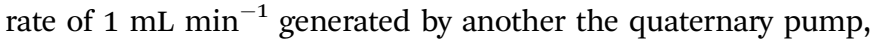
while the pretreatment column is simultaneously washed by $100 \%$ acetonitrile to remove matrices, and the matrices were sent to waste (Fig. 1C). After the analytes analysis was finished, the IC system kept at the initial state (Fig. 1A) to achieve the equilibrium for the next sample. The detailed procedures were described in the Table 1.

$\mathbf{A}$

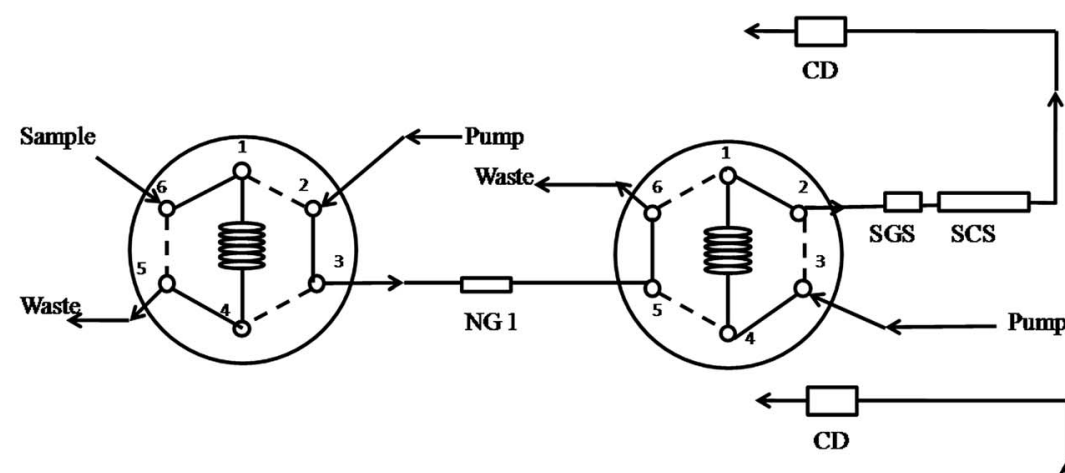

B
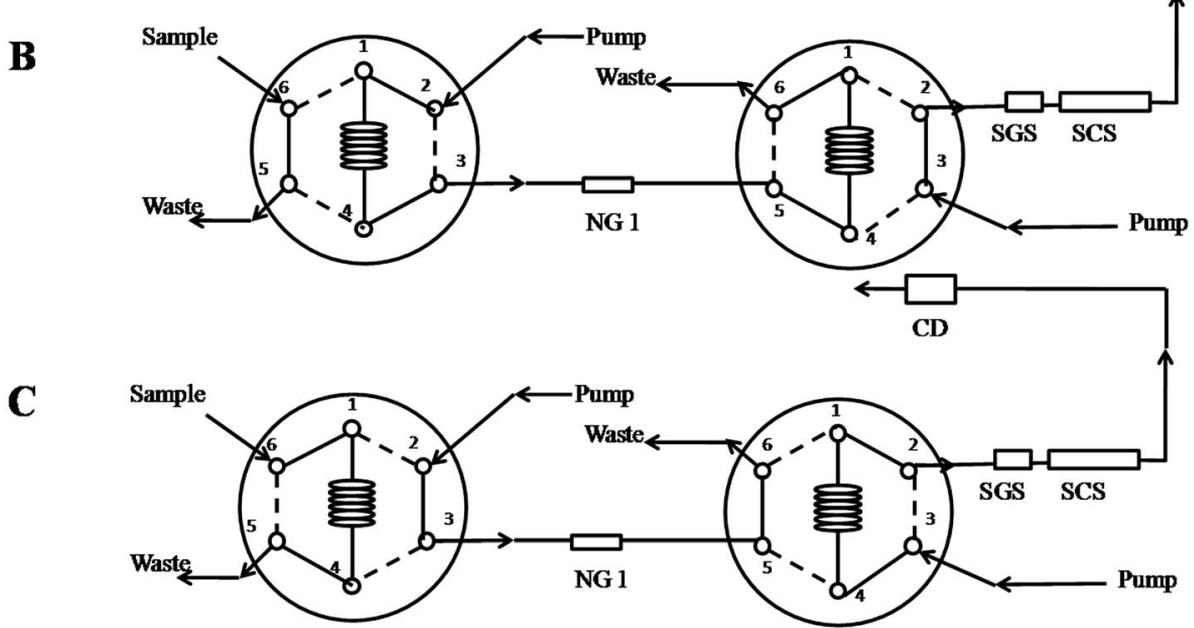

Fig. 1 Chromatographic instrument configuration for the column-switching system. (A) Filling the sample loop; (B) on-line sample pretreatment; (C) analyzing six analytes and washing the pretreatment column. 
Table 1 System operation procedure

\begin{tabular}{|c|c|c|c|c|c|}
\hline Procedure & Time (min) & $\begin{array}{l}\text { Elution of the } \\
\text { pretreatment column }\end{array}$ & Elution of the analytical column & Valve 1 & Valve 2 \\
\hline (1) Sampling & & $3 \mathrm{mmol} \mathrm{L}^{-1} \mathrm{MSA}$ & $3 \mathrm{mmol} \mathrm{L}{ }^{-1} \mathrm{MSA}+10 \%$ acetonitrile & Load & Inject \\
\hline (3) Analyzing and rinsing of NG1 & $0.5-10.5$ & Acetonitrile & $3 \mathrm{mmol} \mathrm{L}{ }^{-1} \mathrm{MSA}+10 \%$ acetonitrile & Load & Inject \\
\hline $\begin{array}{l}\text { (4) Analyzing and NG1 achieving the } \\
\text { equilibrium }\end{array}$ & $10.5-20$ & $3 \mathrm{mmol} \mathrm{L}^{-1} \mathrm{MSA}$ & $3 \mathrm{mmol} \mathrm{L}{ }^{-1} \mathrm{MSA}+10 \%$ acetonitrile & Load & Inject \\
\hline
\end{tabular}

\section{Results and discussion}

\subsection{Optimization of chromatographic conditions}

Prior to the column-switching IC analysis, minimal samplepreparation steps, such as centrifugation or filtration through $0.45 \mu \mathrm{m}$ filters, are recommended to prolong the lives of the pretreatment column and the analytical column. Centrifuged or filtered samples have micro particles removed as much as possible to avoid plugging the system. Therefore, minimal procedures (proteins precipitation, centrifugation and filtration) are needed prior to column-switching IC system for simultaneous determination of L-carnitine, choline and mineral ions in milk products.

Sodium, potassium, calcium and magnesium are the main cations in milk. L-Carnitine and choline are highly polar and hydrophilic compounds, and can be converted into cations on acidic conditions. Thus, it is easy to exclude these ionic molecules on the ion excluding column of Dionex IonPac NG1, while water-solvable organic matrixes still remaining in samples can be retained in IonPac NG1 column. Due to the distinct physicochemical characteristics of the substances, ion excluding column (Dionex IonPac NG1) for pretreatment and ion exchange columns (Dionex IonPac SCS and IonPac SGS column) for separation were used in method development.

The column-switching IC method was optimized by changing the analytical conditions in order to obtain the optimal chromatographic performances. The conditions evaluated include the mobile phase of pretreatment column and analytical column, column-switching time and effects of possible matrix ions. All optimization studies were carried out in triplicates.

3.1.1 Optimization of mobile phase of the analytical column. The chromatographic conditions optimization was primarily focused on selection of a stationary phase, which was able to separate the target cations using suitable mobile phases. Among several different columns tested (IonPac CS 12A, IonPac CS 17, IonPac CS 16), best performances in terms of retention, separation and detection were achieved on IonPac SCS. MSA has been selected in this study because of its relative low conductivity. ${ }^{21}$ Different concentrations of MSA ranging from 3 to $5 \mathrm{mmol} \mathrm{L}{ }^{-1}$ have been studied to determine the optimum concentration, with an injection volume of $25 \mu \mathrm{L}$ and using nonsuppressed conductivity detection. The result showed that the separation of analytes and background conductivity were worse at the higher concentration, and the low concentration resulted in broad peak shape. A faster speed resulted in peaks overlap, while the lower the velocity was, the longer the overall time was, as well as the worse peak shape. Thus, it is suggested to use

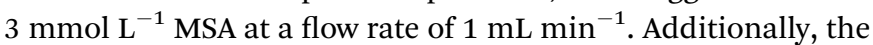
addition of acetonitrile into MSA provided better separation performance of the analytes (shorter separation time and lower background conductivity) than no organic solvent added. The ratio of acetonitrile (v/v) to $3 \mathrm{mmol} \mathrm{L}^{-1}$ MSA was varied from $5 \%$ to $20 \%$. Thus, $10 \%(\mathrm{v} / \mathrm{v})$ acetonitrile was selected for the following experiment (Fig. S2 $\dagger$ ). Considering retention time, peak shape and detector sensitivity, a mobile phase $3 \mathrm{mmol} \mathrm{L}^{-1}$ MSA with acetonitrile concentration of $10 \%(\mathrm{v} / \mathrm{v})$ was selected. Fig. 4D shows the chromatogram of a standard solution.

3.1.2 Optimization of the pretreatment column. The method for the rapid on-line separation of target cations from organic matrix using short ion excluding column Dionex IonPac NG1 was developed. The conditions affecting the clean-up performance of the pretreatment column were optimized in order to eliminate the water-soluble or acetonitrile-soluble organic interferences still remaining in milk samples. It turned out that the cationic forms of the analytes with MSA as elution can be easily separated from organic matrix using short the pretreatment column and the concentrations of MSA have little influence on the retention behavior. Thus, $3 \mathrm{mmol} \mathrm{L}^{-1}$ MSA is also selected as elution of the pretreatment column. Fig. 2 showed the elimination performance of the pretreatment column with $3 \mathrm{mmol} \mathrm{L}^{-1} \mathrm{MSA}$ as the eluent at different flow rate of $0.5 \mathrm{~mL} \mathrm{~min}{ }^{-1}, 1.0 \mathrm{~mL} \mathrm{~min}^{-1}$ and $1.5 \mathrm{~mL} \mathrm{~min}^{-1}$. The analysis was carried out with a six-port valve, an IonPac NG1 column and an UV detector working at the wavelength ranging from 200 to $400 \mathrm{~nm}$. The results showed that a flow rate of $1.0 \mathrm{~mL} \mathrm{~min}^{-1}$ was optimum for the pretreatment column. As the flow rate increased, the increase of absorbance in the range of 200 to $400 \mathrm{~nm}$ indicated the sorbent traps the less organic matrices and most of them were eluted immediately, while the decrease to $0.5 \mathrm{~mL} \mathrm{~min}^{-1}$ could not significantly improve organic component interferences but lead to the longer analytical time.

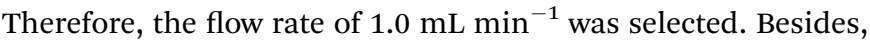
most matrices of milk powder sample were also removed by online pretreatment system at the flow rate of $1.0 \mathrm{~mL} \mathrm{~min} \mathrm{~m}^{-1}$ (Fig. S1†). Then the pretreatment column is washed with a mobile phase of an eluotropic strength to wash the organic matrices remaining in the IonPac NG1 column.

3.1.3 Optimization of column-switching time. Subsequently, the column-switching time was optimized to ensure cations were collected completely. After sample was injected, it was delivered from the pretreatment column directly into the 
A

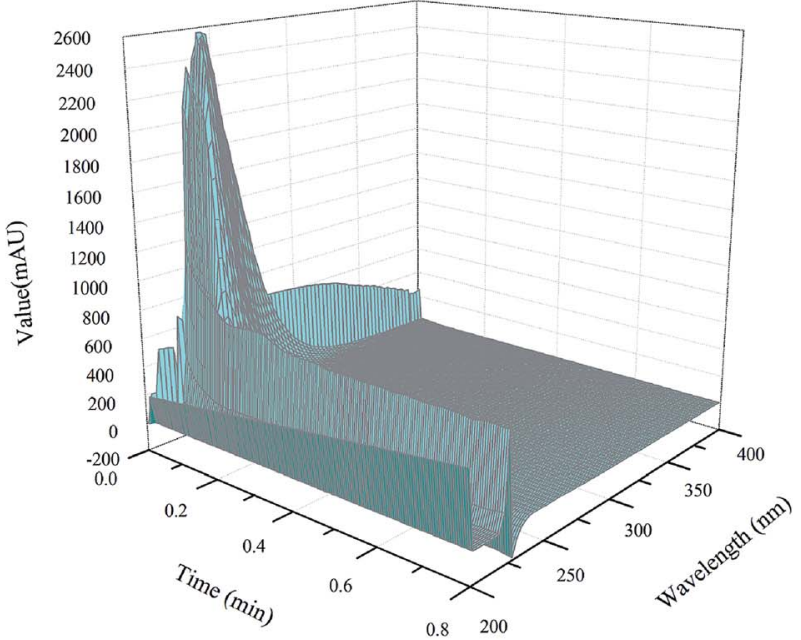

C

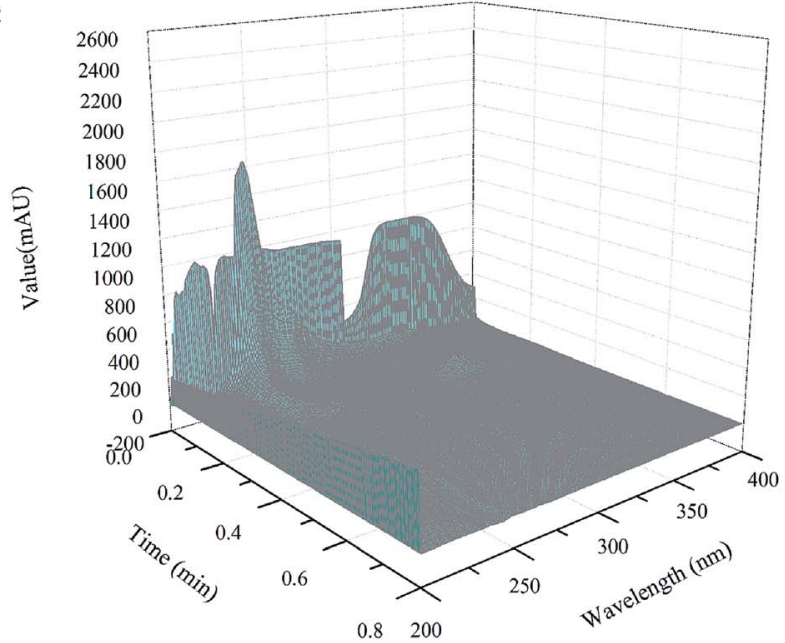

B

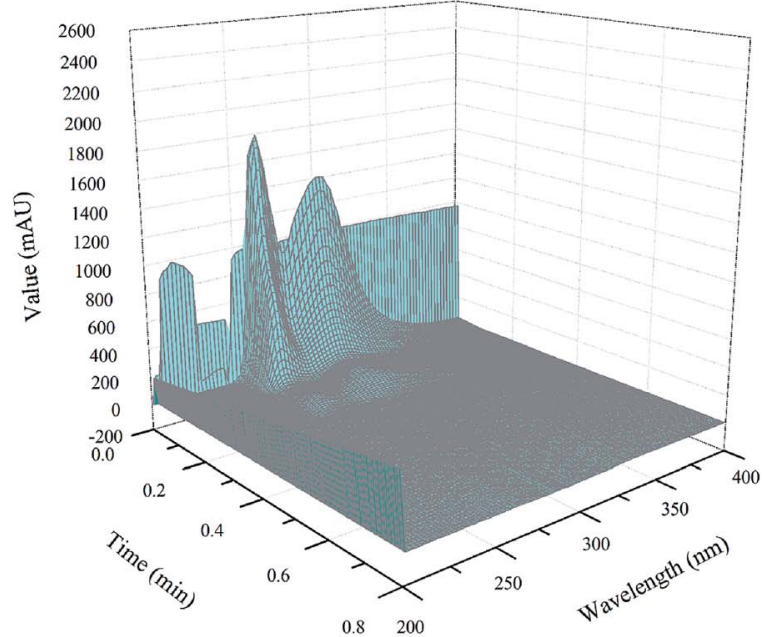

D

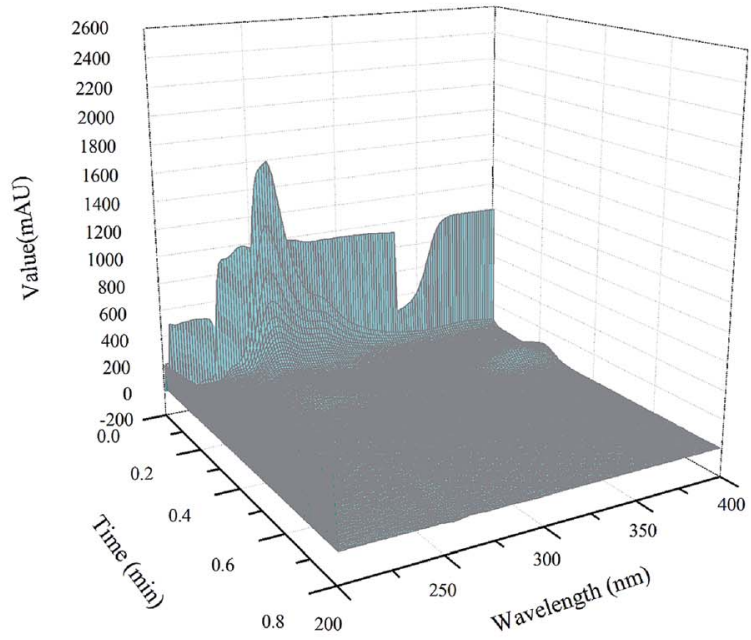

Fig. 2 Three dimensional diagram of the real sample, analysis of milk sample by direct injection at the flow rate of $1 \mathrm{~mL} \mathrm{~min}^{-1}$ (A), analysis of milk sample by on-line pretreatment method at the flow rate of $1.5 \mathrm{~mL} \mathrm{~min}^{-1}$ (B), $1 \mathrm{~mL} \mathrm{~min}^{-1}$ (C) and $0.5 \mathrm{~mL} \mathrm{~min}^{-1}$ (D). Injection volume: $25 \mu \mathrm{L}$; UV detection at $200-400 \mathrm{~nm}$, mobile phase: $3 \mathrm{mmol} \mathrm{L}^{-1} \mathrm{MSA}$.

conductivity detector with non-suppressed conductivity detection. Within $0.5 \mathrm{~min}$, the analytes were all washed out Fig. 3. Additionally, Fig. 2 also showed that most matrices were removed from the samples. Therefore, the switching time of the valve 2 was set at $0.5 \mathrm{~min}$ to ensure all the analytes were washed out at $1 \mathrm{~mL} \mathrm{~min}^{-1}$.

\subsection{Method validation}

To evaluate the proposed method, experiments with regard to the linearity, limit of detection (LOD), limit of quantification (LOQ), precision selectivity and robustness of the method were performed.

3.2.1 Linearity, LOD and LOQ. The calibration standard solutions were used for establishment of calibration curve. Three replicate IC column-switching determinations were performed for each level under the optimized experimental conditions. As shown in Table 2, the linearity was excellent with correlation coefficients $\left(r^{2}\right)$ greater than 0.997 in the concentration range of $1-100 \mathrm{mg} \mathrm{L}^{-1}$. The detection limits (LOD) (the

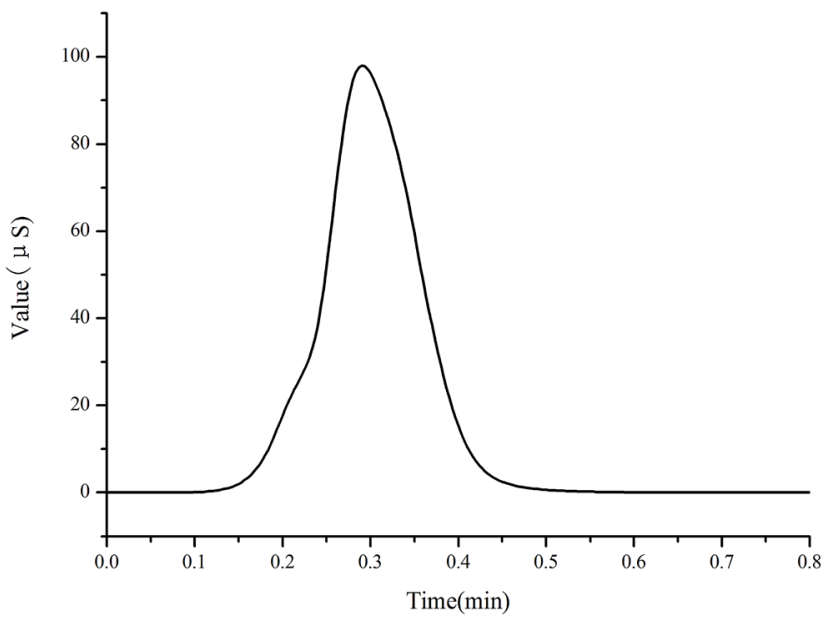

Fig. 3 Chromatogram of standard solution (15 mg L ${ }^{-1}$ sodium, potassium, magnesium, calcium; $10 \mathrm{mg} \mathrm{L}^{-1} \mathrm{~L}$-carnitine, choline) by the

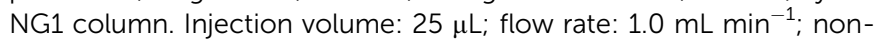
suppressed conductivity detector, mobile phase: $3 \mathrm{mmol} \mathrm{L}^{-1} \mathrm{MSA}$. 
Table 2 Summary of linearity, LOD and LOQ data

\begin{tabular}{|c|c|c|c|c|c|c|c|}
\hline Analyte & Linear equation & $r^{2}$ & Linear $\left(\mathrm{mg} \mathrm{L}^{-1}\right)$ & $\operatorname{LOD}\left(\mathrm{mg} \mathrm{L}^{-1}\right)$ & $\mathrm{LOQ}\left(\mathrm{mg} \mathrm{L}^{-1}\right)$ & \multicolumn{2}{|c|}{$\operatorname{RSD}(n=5) \%$} \\
\hline $\mathrm{K}$ & $y=0.0030 x+0.0027$ & 0.9976 & $1-100$ & 0.15 & 0.50 & 0.9 & 0.4 \\
\hline L-Carnitine & $y=0.0120 x+0.0003$ & 0.9962 & $2-200$ & 0.25 & 0.82 & 0.9 & 0.7 \\
\hline Choline & $y=0.0124 x+0.0373$ & 0.9954 & $1-100$ & 0.10 & 0.35 & 1.2 & 0.6 \\
\hline
\end{tabular}

Table 3 Analytes recovery and repeatability data from infant milk, milk powder, milk $(n=5)$

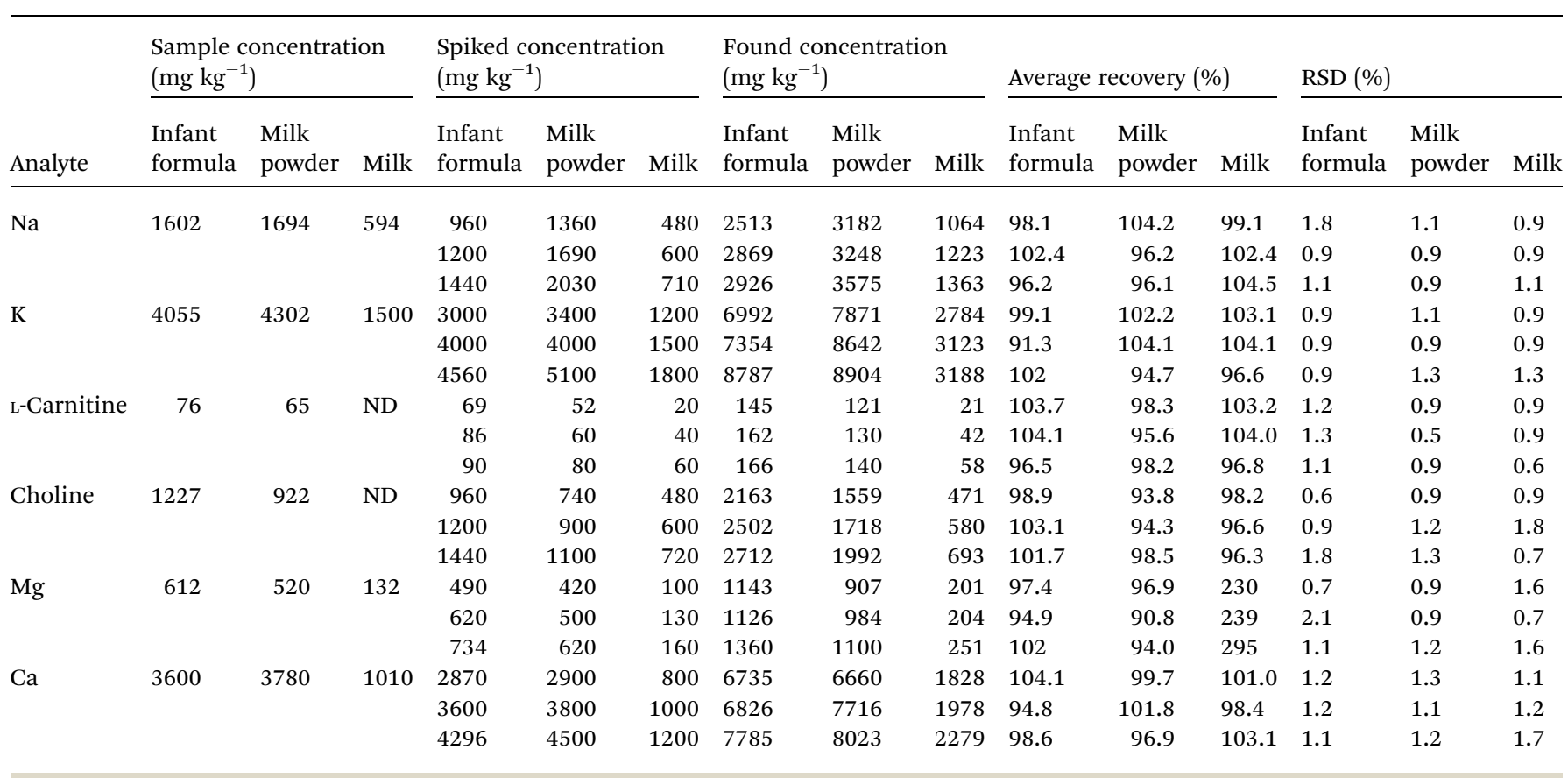

lowest concentration yielding a signal-to-noise of 3) and the limit of quantification (LOQ) (signal-to-noise ratio of 10) were also calculated. The LOD and LOQ were determined by serial dilution of real sample solutions using the established IC column-switching method until the signal-to-noise at $3: 1$ and $10: 1$ respectively. The values were shown in Table 2 .

3.2.2 Repeatability, accuracy and precision. The repeatability was investigated by measuring RSD for peak area and retention time. Sample was injected into the column-switching IC system $(n=5)$. Replicate data exhibited acceptable precision (relative standard deviation, RSD $\leq 2 \%$ ). Table 2 shows the summary results.

Accuracy and precision were determined by recovery test using spiked purchased milk samples. The non-spiked samples and fortified samples with three concentration levels were subjected to the entire sample pretreatment, column-switching procedures and analytical sequences. All determinations were performed in five times and the means of the results were used. The results are summarized in the Table 3. The average recoveries ranged from $94 \%$ to $105 \%$ for the proposed method, with the RSD below $3 \%(n=5)$. The chromatograms of spiked real

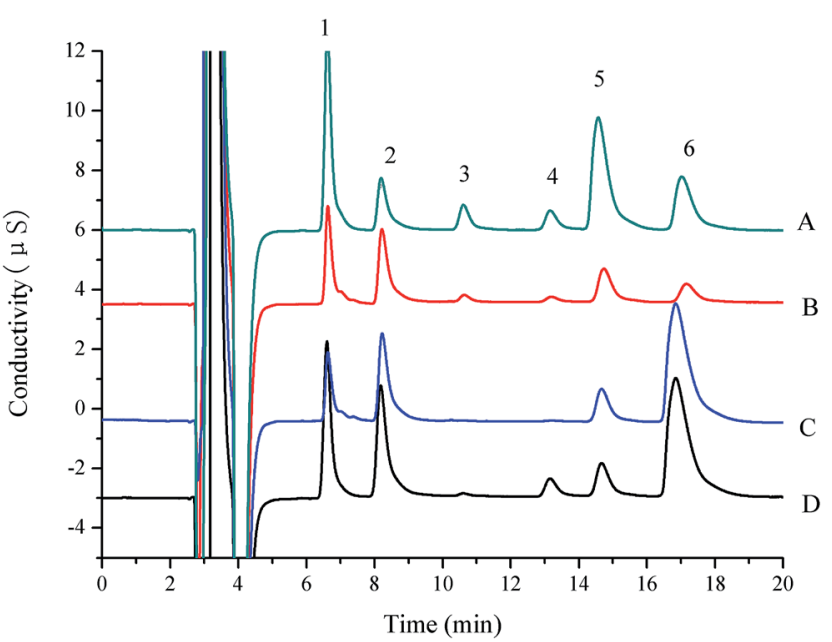

Fig. 4 Chromatograms of standard solution (15 $\mathrm{mg} \mathrm{L}^{-1}$ sodium, potassium, magnesium, calcium; $10 \mathrm{mg} \mathrm{L}^{-1} \mathrm{~L}$-carnitine, choline) (A), infant formula (B), milk (C) and milk powder (D); peaks: (1) sodium; (2) potassium; (3) L-carnitine; (4) choline; (5) magnesium; (6) calcium. 
Table 4 Determination of analytes content in infant formula, milk powder, milk samples $(n=5)$

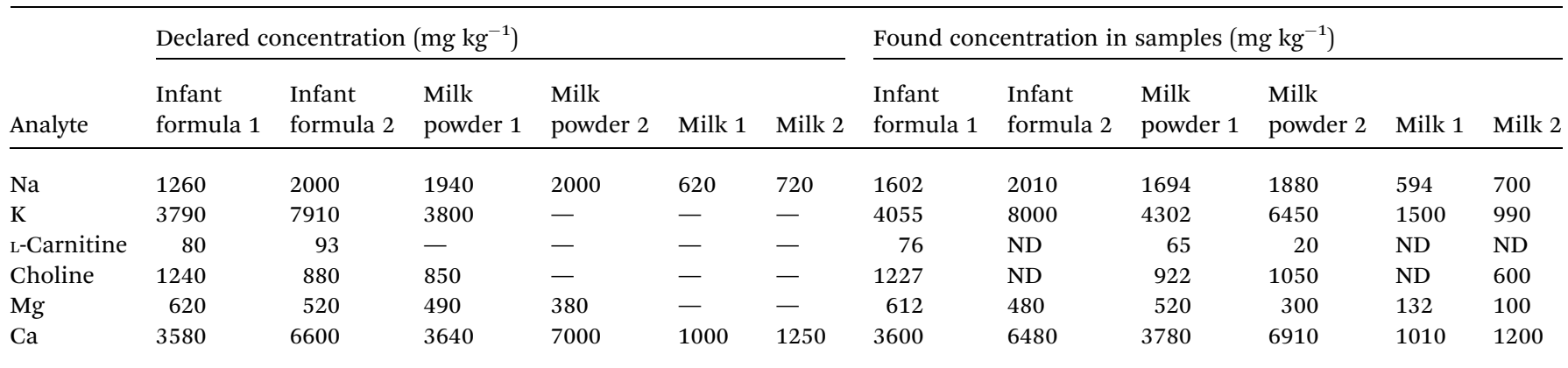

samples by the column-switching IC procedure are shown in Fig. 4 . The accurate determination of $\mathrm{L}$-carnitine, choline and sodium, potassium, magnesium and calcium ions in milk and powdered infant formula can be attained by this method.

3.2.3 Robustness of the method. To evaluate the robustness of the method, the effect of deliberated and small changes on the retention time and peak area was measured. Method parameters like concentration of acetonitrile (range: 9 and 11\% $\mathrm{v} / \mathrm{v}$ ), concentration of MSA (range: 2.9 and $3.1 \mathrm{mmol} \mathrm{L}^{-1}$ ) in second chromatography dimension, temperature in column oven (range: 25 and $35{ }^{\circ} \mathrm{C}$ ) and mobile phase flow rate (range: 0.9 and $1.1 \mathrm{~mL} \mathrm{~min}^{-1}$ ) were taken into consideration. At a time only one parameter was changed while the other factors have not altered. The relative standard deviations of peak area of each analyte were compared with peak area under optimized conditions. High variations in retention time and peak area over $8 \%$ due to the changes in concentration of acetonitrile, column oven temperature indicated such optimized conditions of the method must be strictly kept. As expected, the mobile phase flow rate demonstrated the least influence on the system suitability parameters while factors like concentration of MSA in second chromatography dimension have altered the retention times and peak less than $2 \%$. Low variations of peak area could demonstrate that the optimized and validated conditions of the method are robust and small changes in the mobile phase flow rate and concentration of MSA in second chromatography will not affect results of analysis.

\subsection{Application of the method}

To verify the applicability of the proposed method, determination of these nutrients in six milk products from different local supermarkets was conducted. The chromatogram of target analytes obtained from milk samples was shown in Fig. 3, and the compositional data of six milk samples was tabulated in Table 4. Peak purity in sample run showed that no interference was observed in the quantitative analysis of six analytes. The suggested procedure of sample preparation was simple, fast and reliable. Much of the nutrients contents measured by the proposed method had values close to label declared. It was clear that the predominant components were potassium and calcium, which up to $60 \%$ of all the quantitative analytes. It has been showed that concentrations of the minerals have no statistically significant differences from the label claims. Surprisingly, without mentioned in the label claim, L-carnitine was detected in milk powder 2 and choline was detected in milk 1 . It may be that natural milk contains $\mathrm{L}^{-}$ carnitine and choline.

\section{Conclusions}

In this paper, a simple and automated column-switching IC method with non-suppressed conductivity detection has been developed for the simultaneous determination of L-carnitine, choline, mineral ions (free sodium, potassium, magnesium, calcium ions) in infant formula, milk powder and milk. The developed method proved to be simple, efficient and reliable and may have the large potential applications in measurement and assessment of the nutritional quality of milk products.

\section{Acknowledgements}

This research was financially supported by Key Laboratory of Health Risk Appraisal for Trace Toxic Chemicals of Zhejiang Province, Science and Technique Plan of Zhejiang TCM (No. 2016ZQ003), Zhejiang Provincial Health and Medicinal Science Foundation (2016RCB003) and Zhejiang Provincial Natural Science Foundation of China (No. Y4110532, LY12B05003, LY13B020001, LQ13B050001).

\section{References}

1 F. A. R. Martino, M. L. F. Sanchez and A. S. Medel, J. Anal. At. Spectrom., 2000, 15, 163-168.

2 F. Masotti, D. Erba, I. De Noni and L. Pellegrino, J. Dairy Sci., 2012, 95, 2872-2881.

3 I. Nadaoka, E. Hatakeyama, C. Tanada, T. Sakamoto, S. Fukaya, T. Akiba, K. Inoue, Y. Yamano and T. Toyo'oka, Anal. Sci., 2014, 30, 839-844.

4 Y. P. Xiong, Y. Y. Zhao and S. Goruk, J. Chrom. B, 2012, 911, 170-179.

5 S. H. Zeisel, Nutrition, 2000, 16, 669-671.

6 J. R. Hartwell, M. J. Cecava and S. S. Donkin, J. Dairy Sci., 2000, 83, 2907-2917.

7 J. Sales, P. Homolka and V. Koukolová, J. Dairy Sci., 2010, 93, 3746-3754. 
8 R. Gao, E. J. M. Temminghoff, H. P. van Leeuwen, H. J. F. van Valenberg, M. D. Eisner and M. A. J. van Boekel, Int. Dairy J., 2009, 19, 431-436.

9 L. Noël, M. Carl, C. Vastel and T. Guérin, Int. Dairy J., 2008, 18, 899-904.

10 G. Yellen, Nature, 2002, 419, 35-42.

11 M. C. Latham, Human nutrition in the developing world, Popul. Dev. Rev., 1998, 24, 177.

12 S. H. Zeisel and K. A. Dacosta, J. Nutr. Biochem., 1990, 1, 5559.

13 P. Donato, F. Cacciola, F. Cichello, M. Russo, P. Dugo and L. Mondello, J. Chromatogr. A, 2011, 1218, 6476-6482.

14 N. Tyrefors and A. Carlsson, J. Chromatogr. A, 1990, 502, 337349.

15 S. H. Chen, V. Soneji and J. Webster, J. Chromatogr. A, 1996, 739, 351-357.

16 K. Oates, L. Chen, B. De Borba, D. Mohindra, J. Rohrer and D. Dowell, J. AOAC Int., 2013, 96, 1400-1406.
17 D. C. Suo, L. Li, S. Zhang and X. O. Su, Anal. Methods, 2013, 5, 59-63.

18 L. B. de Caland, E. L. C. Silveira and M. Tubino, Anal. Chim. Acta, 2012, 718, 116-120.

19 J. M. Liu, C. C. Liu, G. Z. Fang and S. Wang, RSC Adv., 2015, 5, 58713-58726.

20 M. Laikhtman and J. S. Rohrer, J. AOAC Int., 1999, 82, 11561162.

21 J. J. Zhang and Y. Zhu, J. Chromatogr. A, 2007, 1170, 114-117. 22 A. Azzouz, A. J. Rascón and E. Ballesteros, J. Pharm. Biomed. Anal., 2016, 119, 16-26.

23 L. Campone, A. L. Piccinelli, R. Celano, I. Pagano, M. Russo and L. Rastrelli, J. Chromatogr. A, 2016, 1428, 212-219.

24 B. Ivana, H. Markéta, Š. Dalibor and S. Petr, Food Chem., 2013, 141, 1433-1437.

25 C. Z. Ni, B. H. Zhu, N. N. Wang, M. H. Wang, S. Q. Chen, J. J. Zhang and Y. Zhu, Food Chem., 2016, 194, 555-560.

26 Z. Zhu, L. Xi, Q. Subhani, Z. Huang and Y. Zhu, Talanta, 2013, 113, 113-117. 